\title{
From light to mass: accessing the initial and present-day Galactic globular cluster mass functions
}

\author{
C. Bonatto ${ }^{\star}$ and E. Bica \\ Departamento de Astronomia, Universidade Federal do Rio Grande do Sul, Av. Bento Gonçalves, 9500 Porto Alegre 91501-970, RS, Brazil
}

Accepted 2012 March 20. Received 2012 March 7; in original form 2012 January 24

\begin{abstract}
The initial and present-day mass functions (ICMFs and PDMFs, respectively) of the Galactic globular clusters (GCs) are constructed based on their observed luminosities, the stellar evolution and dynamical mass-loss processes, and the mass-to-light ratio $(M / L)$. Under these conditions, a Schechter-like ICMF is evolved for approximately a Hubble time and converted into a luminosity function (LF), which requires finding the values of five free parameters: the mean GC age $\left(\bar{t}_{\mathrm{gc}}\right)$, the dissolution time-scale of a $10^{5}-\mathrm{M}_{\odot}$ cluster $\left(\tau_{5}\right)$, the exponential truncation mass $\left(\mathcal{M}_{\mathrm{c}}\right)$ and two $M / L$ parametrizing constants. This is achieved by minimizing the residuals between the evolved and observed LFs, with the minimum residuals and realistic parameters obtained with $M / L$ s that increase with luminosity (or mass). The optimum PMDFs indicate a total stellar mass of $\sim 4 \times 10^{7} \mathrm{M}_{\odot}$ still bound to GCs, representing $\sim 15$ per cent of the mass in clusters at the beginning of the gas-free evolution. The corresponding ICMFs resemble the scale-free MFs of young clusters and molecular clouds observed in the local Universe, while the PDMFs follow closely a lognormal distribution with a turnover at $\mathcal{M}_{\text {to }} \sim 7 \times 10^{4} \mathrm{M}_{\odot}$. For most of the GC mass range, we find an $M / L$ lower than usually adopted, which explains the somewhat low $\mathcal{M}_{\text {to }}$. Our results confirm that the $M / L$ increases with cluster mass (or luminosity), and suggest that GCs and young clusters share a common origin in terms of physical processes related to formation.
\end{abstract}

Key words: globular clusters: general.

\section{INTRODUCTION}

Globular clusters (GCs) are the living fossils of an epoch dating back to the time of the Milky Way's formation and thus they probably share the same physical conditions and processes as that originated our Galaxy. Since formation, GCs are continuously affected by several sources of mass loss, but, because of a large initial mass and/or an orbit that takes them most of the time away from the Galactic Centre and/or disc, they are, in general, characterized by a long longevity. This suggests that some primordial information may have been preserved, if not at an individual scale, at least with respect to the collective properties shared by the surviving GC population.

In this context, an interesting issue is the origin of the Milky Way's (old) GC population and its relation to the young massive clusters that form in starbursts and galaxy mergers of the local Universe. If both old and young cluster populations share a similar, starburst origin, then the mass distribution of their members should also be characterized by similar mass functions (MFs), something that is ruled out by observations in several local galaxies. For

\footnotetext{
${ }^{\star}$ E-mail: charles.bonatto@ufrgs.br
}

instance, when expressed in terms of the number of GCs per unit logarithmic mass interval $(\mathrm{d} N / \mathrm{d} \log \mathcal{M})$, the MFs of young clusters in several galaxies follow monotonically a power law of slope $\approx-1$, while the present-day MF (PDMF) of GCs presents a turnover for masses lower than $\mathcal{M}_{\mathrm{to}} \sim 10^{5} \mathrm{M}_{\odot}($ McLaughlin \& Fall 2008, and references therein). The low-mass drop in the lognormal PDMF has been interpreted as mainly due to the large-scale dissolution of low-mass GCs after several Gyr of tidal interactions with the Galaxy and internal dynamical processes (e.g. Kruijssen 2008; McLaughlin \& Fall 2008; Kruijssen \& Portegies Zwart 2009).

Consequently, since mass loss continuously changes the MF shape - especially in the low-mass range - a direct comparison of several Gyr old PDMFs with the MF of young clusters should not be used to draw conclusions on a physical relation between both populations. On the other hand, this raises another, but related question of whether or not the initial MFs (ICMFs) of globular and young clusters are similar, since theoretical evidence suggests that cluster formation leaves distinct imprints in the ICMFs (Elmegreen $\&$ Falgarone 1996). Thus, if both cluster populations have the same origin, this should be reflected in the respective ICMFs.

Given the above, the reconstruction of the Milky Way's GC ICMF requires having access not only to the (as complete as possible) PDMF, but also to the mass-loss physics. As for the latter, recent 
numerical simulations and semi-analytical models have produced robust relations for the mass evolution of star clusters located in different environments and losing mass through stellar evolution and tidal interactions with Galactic substructures and giant molecular clouds (e.g. Lamers et al. 2005; Lamers, Baumgardt \& Gieles 2010).

Over the years, the GCMF time evolution has been investigated with a wide variety of approaches. For instance, Vesperini (1998) takes the effects of stellar evolution, two-body relaxation, disc shocking, dynamical friction, and the location in the Galaxy into account to follow the evolution of individual GCs. The main findings are as follows: (i) the large-scale disruption of GCs in the central parts of the Galaxy leads to a flattening in their spatial distribution; (ii) if the initial GCMF shape is lognormal, it is preserved in most cases; and (iii) in the case of an initial power-law GCMF, evolutionary processes tend to change it into lognormal. Baumgardt (1998) considers GC systems formed with a power-law MF and follows the orbits of individual clusters. Taking several GC destruction mechanisms into account, he finds luminosity distributions that depend on the Galactocentric distance. Vesperini et al. (2003) study the dynamical evolution of M87 GCs with numerical simulations that cover a range of initial conditions for the GCMF and for the spatial and velocity distribution of the GCs. The simulations include the effects of two-body relaxation, dynamical friction, and stellar evolutionrelated mass loss. They confirm that an initial power-law GCMF can be changed into bell shaped through dynamical processes, and show that the inner flattening observed in the spatial distribution of M87 GCs can result from the dynamical evolution on an initially steep density profile. Parmentier \& Gilmore (2007) show that an approximately Gaussian MF is naturally generated from a powerlaw mass distribution (but with a lower mass limit) of protoglobular clouds by expulsion from the protocluster of star-forming gas due to supernova activity. Shin, Kim \& Takahashi (2008) target the MF and radial distribution evolution of the Galactic GCs by means of a Fokker-Planck model that considers dynamical friction, disc/bulge shocking and eccentric GC orbits. Their best-fitting models predict the initial GC mass to be in the range $M_{\mathrm{GC}}^{0} \sim(1.5-1.8) \times 10^{8} \mathrm{M}_{\odot}$.

At some point, most of the previous works compare models with the observed GC PDMF. However, especially because of the large distances involved, a direct determination of the stellar mass for most GCs is not available, which means that the observational PDMF cannot be taken as an ideal starting point for this analysis. Instead, the most direct and robust observable available for the present-day Milky Way's GC population as a whole is the luminosity function (LF), which is usually expressed in terms of the absolute $V$ magnitude. Obviously, the use of luminosity requires a mass-to-light ratio $(M / L)$ to convert LFs into MFs.

Recent observational and theoretical findings have convincingly shown that $M / L$ does not remain constant with respect to cluster mass. Instead, the dynamical evolution of clusters - and the preferential loss of low-mass stars - also affects the $M / L$. Specifically, for a given age and metallicity, the $M / L$ is expected to increase with cluster mass (or luminosity; e.g. Kruijssen 2008; Kruijssen \& Portegies Zwart 2009). Interestingly, the $M / L$ dependence on luminosity (or mass) is already suggested by the dynamical cluster mass and $M / L$ estimates of Pryor \& Meylan (1993).

Our goal in this paper is to reconstruct both the ICMF and the PDMF of the Galactic GCs, starting with their observed LF and taking the mass loss into account. In this process, different analytical forms of the $M / L$ will be tested to search for constraints.

This paper is organized as follows. In Section 2, we build the observed and theoretical LFs of the Galactic GCs, convert the latter into the PDMF, and discuss the adopted M/Ls. In Section 3, we briefly describe the parameter-search method. In Section 4, we discuss the results. Concluding remarks are given in Section 5.

\section{THE THEORETICAL PRESENT-DAY LF}

The first step in our analysis is to build the observed (or present-day) LF $\left[\phi\left(M_{V}\right) \equiv \mathrm{d} N / \mathrm{d} M_{V}\right.$, that is, the number of GCs per unit absolute magnitude interval] of the Galactic GCs. The data were taken from the 2010 revision of the catalogue compiled by Harris (1996), which contains 156 GCs with bona fide absolute magnitudes in the $V$ band $\left(M_{V}\right)$. By definition, the integral of $\phi\left(M_{V}\right)$ over the full $M_{V}$ range is the input number of GCs. As a caveat, we note that several works indicate that not all Galactic GCs share a common history (e.g. Bica et al. 2006, and references therein), with some having been accreted from dwarf galaxies (e.g. Hilker \& Richtler 2000; Parmentier et al. 2000) and others associated with the Galactic thick disc (e.g. Zinn 1985).

Following previous work (e.g. Bonatto \& Bica 2011), uncertainties in $M_{V}$ are incorporated in the LF by assuming a normal (Gaussian) distribution of errors. Specifically, if the magnitude and standard deviation of a GC (usually, the mean value over a series of independent measurements) are given by $\bar{M}_{V} \pm \sigma$, then the probability of finding it at a specific value $\mathrm{M}_{\mathrm{V}}$ is given by $P\left(M_{V}\right)=\frac{1}{\sqrt{2 \pi} \sigma} \mathrm{e}^{-\frac{1}{2}\left(\frac{M_{V}-\bar{M}_{V}}{\sigma}\right)^{2}}$. Next, we build a grid of bins covering the full $M_{V}$ range in Harris (1996), and, for each bin, we sum the $M_{V}$ density (or probability) for all GCs (i.e. the difference between the error functions computed at the bin borders). However, since uncertainties are not given in Harris (1996), for simplicity, we adopt relative errors that depend on the apparent magnitude $\left(m_{V}\right)$ as $\epsilon=0.1 \mathrm{e}^{\left(m_{V} / 27\right)}$. Thus, relatively bright $\left(m_{V} \lesssim 10\right)$ GCs have $\epsilon \lesssim 0.15$, while fainter ones $\left(m_{V} \gtrsim 20\right)$ have $\epsilon \gtrsim 0.2$. Finally, we assume that the absolute error in $M_{V}$ is simply given by $\sigma=\epsilon \times$ $\left|M_{V}\right|$. As shown in Bonatto \& Bica (2011), the end result is a distribution function significantly smoother than a classical histogram. Indeed, the observed LF (Fig. 1) is approximately Gaussian shaped with a pronounced peak around $M_{V} \approx-7$ and $\approx 4$ mag of full width at half-maximum.

As usual, with respect to distribution functions, the observed $\phi\left(M_{V}\right)$ can be linked to the PDMF, $\xi(\mathcal{M}) \equiv \mathrm{d} N / \mathrm{d} \mathcal{M}$, by

$\phi\left(M_{V}\right)=\xi(\mathcal{M})\left|\frac{\mathrm{d} \mathcal{M}}{\mathrm{d} M_{V}}\right|$.

However, except for a few cases, the stellar mass of most GCs has not been directly measured, and so the actual shape of $\xi(\mathcal{M})$ remains uncertain. On the other hand, $\xi(\mathcal{M})$ can be inferred if one knows the initial ${ }^{1} \mathrm{MF}$, the GC age, and the mass-loss physics. Formally, if $\xi_{0}\left(\mathcal{M}_{\mathrm{o}}\right)$ is the MF at $t_{\mathrm{o}}$ - and assuming that GCs are not formed subsequently - the MF at a later time $t$ is given by

$\xi(\mathcal{M}, t)=\xi_{\mathrm{o}}\left(\mathcal{M}_{\mathrm{o}}\right)\left|\frac{\partial \mathcal{M}_{\mathrm{o}}}{\partial \mathcal{M}}\right|$

where $\mathcal{M}=\mathcal{M}\left(\mathcal{M}_{\mathrm{o}}, t\right)$, and $\mathcal{M}_{\mathrm{o}}$ represents the individual $\mathrm{GC}$ masses at $t_{0}$.

Several works (e.g. Larsen 2009; Portegies Zwart, McMillan \& Gieles 2010) suggest that star clusters form with a mass distribution that follows the truncated power law of Schechter (1976)

$\xi_{\mathrm{o}}\left(\mathcal{M}_{\mathrm{o}}\right)=\mathrm{k}_{\mathrm{s}} \mathcal{M}_{\mathrm{o}}^{-2} \mathrm{e}^{-\left(\mathcal{M}_{\mathrm{o}} / \mathcal{M}_{\mathrm{c}}\right)}$

${ }^{1}$ In the present context, 'initial' refers to the onset of gas-free evolution. Hereafter, we refer to the beginning of this period as $t_{0}$. 


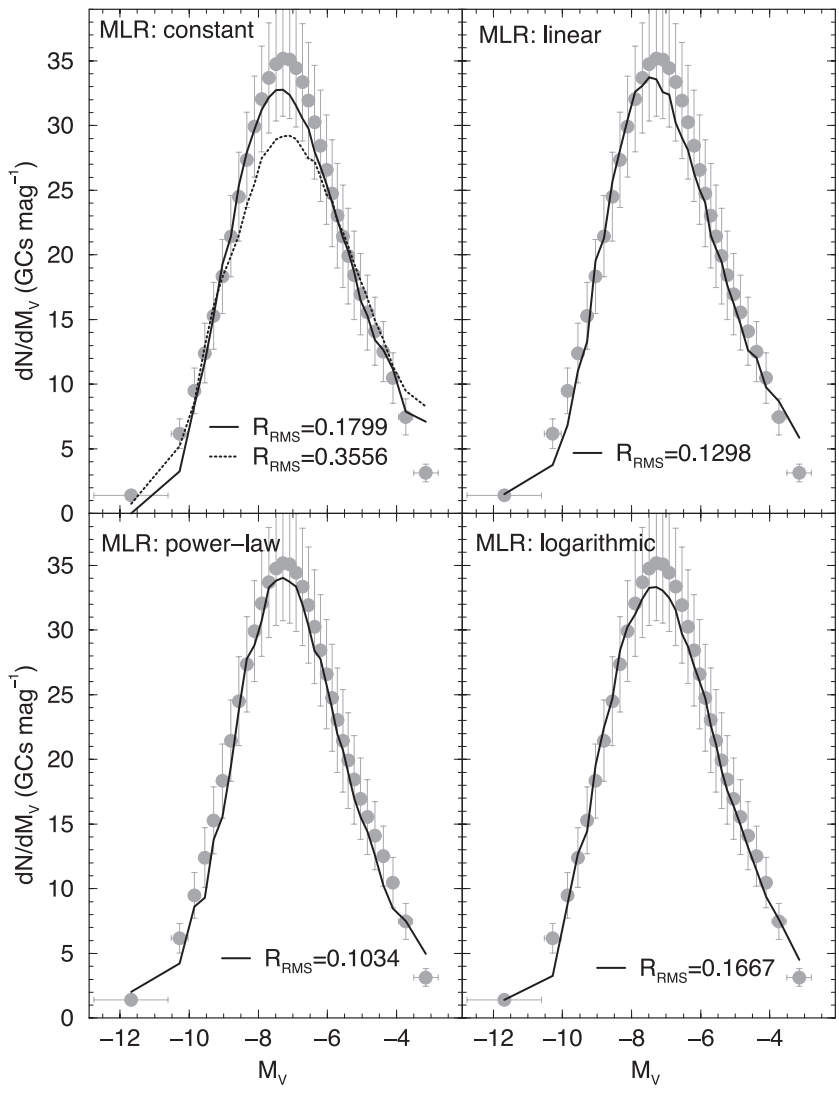

Figure 1. The observed (light-shaded symbols) and best-fitting LFs (solid line) produced with alternative $M / L s$. The respective $R_{\mathrm{rms}}$ are shown. A constant $M / L$ with the mean value of $\mathcal{M} / L_{V}=2$ (dotted line) taken from the literature produces a relatively poor fit with $\mathrm{R}_{\mathrm{rms}} \approx 0.36$ (top left-hand panel).

over the mass range $\mathcal{M}_{\text {inf }}-\mathcal{M}_{\text {sup }}$, where $\mathcal{M}_{\mathrm{c}}$ is the exponential truncation mass, and $k_{\mathrm{s}}$ is a normalization constant. We note that, because of the continuous mass loss (see below), GCs that formed with a relatively low mass may no longer be detected, and can be considered as dissolved. Here, we represent this dissolution limit by the lower observable mass value $\mathcal{M}_{\mathrm{i}}$.

The earliest cluster phase is dominated by effects related to the impulsive removal of the parental, intracluster gas by supernovae and massive-star winds, which rapidly damps cluster formation and changes the ratio between residual gravitational potential and stellar velocity dispersion, thus leading to the first bout of dynamical evolution (e.g. Lada, Margulis \& Dearborn 1984; Goodwin 1997; Geyer \& Burkert 2001; Baumgardt \& Kroupa 2007). Another consequence of these early effects on the ICMF may be a depletion in the number of GCs towards the low-mass range (e.g. Kroupa \& Boily 2002; Baumgardt, Kroupa \& Parmentier 2008; Parmentier et al. 2008). Clusters can be considered to be essentially gas free after the first $t_{\mathrm{o}} \sim 10^{7} \mathrm{yr}$, but they keep losing mass mainly through stellar evolution and externally driven tidal effects. After several Gyr, these processes may reduce the mass to a fraction of the initial value. Following Lamers et al. (2005), we express the mass remaining (due to stellar evolution and tidal effects) in a given GC (with initial mass $\mathcal{M}_{\mathrm{o}}$ ) at time $t$ by

$\mathcal{M}(t)=\mathcal{M}_{\mathrm{o}}\left[\mu_{\mathrm{se}}^{\gamma}-\frac{\gamma t}{\tau_{5}}\left(\frac{\mathcal{M}_{\mathrm{o}}}{10^{5} \mathrm{M}_{\odot}}\right)^{-\gamma}\right]^{1 / \gamma}$, where $\mu_{\mathrm{se}}$ is the stellar evolution mass loss, $\tau_{5}$ is the dissolution time-scale of a $10^{5}-\mathrm{M}_{\odot}$ star cluster, and $\gamma$ is the power-law exponent that sets the dependence of the cluster disruption time on mass. We note that $\mu_{\mathrm{se}}$ is a function of time, but since we are dealing with GCs with ages between 9 and $13 \mathrm{Gyr}$, we simplify this point by taking here the asymptotic value $\mu_{\mathrm{se}} \approx 0.7$ (Lamers et al. 2005). Useful Milky Way approximations for the other parameters are $\gamma \approx 0.7$ (Lamers et al. 2010) and $\tau_{5} \approx 10^{4} \mathrm{Myr}$ (Kruijssen \& Cooper 2012, and references therein). However, $\tau_{5}$ is expected to present a significant dispersion around its quoted value to account for different GC environments and orbital conditions (Kruijssen \& Cooper 2012). In this sense, we consider $\tau_{5}$ to be a free parameter allowed to vary within a given range (Section 3). Thus, inverting equation (4) to have $\mathcal{M}_{\mathrm{o}}=\mathcal{M}_{\mathrm{o}}(\mathcal{M}(t))$ and coupling equations (2) and (3), the PDMF can be expressed as

$\xi(\mathcal{M}, t)=\mathrm{k}_{\mathrm{s}} \mu_{\mathrm{se}} \mathcal{M}^{\gamma-1}\left(\mathcal{M}^{\gamma}+\beta t\right)^{-(1+1 / \gamma)} \mathrm{e}^{-\frac{\left(\mathcal{M}^{\gamma}+\beta t\right)^{1 / \gamma}}{\mu_{\mathrm{se}} \mathcal{M}_{\mathrm{c}}}}$,

where $\beta \equiv\left(\gamma 10^{5 \gamma}\right) / \tau_{5}$.

Formally, equation (5) applies to an individual cluster having evolved over a time $t$ after $t_{\mathrm{o}}$. However, the underlying idea of our approach is to consider not the individual, but the collective - or average - evolution of the full sample of the Galactic GCs until now. This adds another component to our approach because, instead of a single formation event, the Milky Way GCs present some dispersion in age (e.g. Marín-Franch et al. 2009). In this sense, $t$ should be taken as the mean - or at least the representative - age $\left(\bar{t}_{\mathrm{gc}}\right)$ of the GC population. So, hereafter, we consider $t=\bar{t}_{\mathrm{gc}}$. The constant $k_{\mathrm{s}}$ can be computed by noting that the integral of equation (5) over the minimum observable GC mass, $\mathcal{M}_{\mathrm{i}}$, and the maximum remaining mass at time $\bar{t}_{\mathrm{gc}}, \mathcal{M}_{\mathrm{s}}=\mathcal{M}_{\mathrm{sup}}\left(\bar{t}_{\mathrm{gc}}\right)$, corresponds to the present-day number of GCs

$N_{\mathrm{GC}}=\int_{\mathcal{M}_{\mathrm{i}}}^{\mathcal{M}_{\mathrm{s}}} \xi\left(\mathcal{M}, \bar{t}_{\mathrm{gc}}\right) \mathrm{d} \mathcal{M}$

Changing variables in the integral, equation (6) can be reduced to

$N_{\mathrm{GC}}=\frac{k_{\mathrm{s}}}{\mathcal{M}_{\mathrm{c}}} \int_{\tau_{\mathrm{i}}}^{\tau_{\mathrm{s}}}[\tau \ln (\tau)]^{-2} \mathrm{~d} \tau=\frac{k_{\mathrm{s}}}{\mathcal{M}_{\mathrm{c}}}\left[E 1(\ln (\tau))-\frac{1}{\tau \ln (\tau)}\right]_{\tau_{\mathrm{i}}}^{\tau_{\mathrm{s}}}$,

where $E 1$ is the exponential integral,

$\ln \left(\tau_{\mathrm{i}}\right)=\left(\mathcal{M}_{\mathrm{i}}^{\gamma}+\beta \bar{t}_{\mathrm{gc}}\right)^{1 / \gamma} /\left(\mu_{\mathrm{se}} \mathcal{M}_{\mathrm{c}}\right)$

and

$\ln \left(\tau_{\mathrm{s}}\right)=\left(\mathcal{M}_{\mathrm{s}}^{\gamma}+\beta \bar{t}_{\mathrm{gc}}\right)^{1 / \gamma} /\left(\mu_{\mathrm{se}} \mathcal{M}_{\mathrm{c}}\right)$.

Summarizing, the predicted PDMF (equation 5) can be inserted into equation (1) to build the theoretical $\operatorname{LF} \widetilde{\phi}\left(M_{V}\right)$ and, with the usual relation between luminosity $\left(L_{V}\right)$ and absolute magnitude, $L_{V}=10^{-0.4\left(M_{V}-M_{V}^{\odot}\right)}$, with $M_{V}^{\odot}=4.83$, the theoretical LF is expressed as

$\widetilde{\phi}\left(M_{V}\right)=0.4 \xi\left(\mathcal{M}, \bar{t}_{\mathrm{gc}}\right) \frac{\mathrm{d} \mathcal{M}}{\mathrm{d} \log L_{V}}$.

Subsequently, $\widetilde{\phi}\left(M_{V}\right)$ can be compared with the observed $\phi\left(M_{V}\right)$ to search for the parameters that lead to the best match between both. Note that the present-day GC masses in equation (7) should be expressed as a function of $M_{V}$ (through $L_{V}$ ), which can be done by means of an $M / L\left(M / L \equiv \mathcal{M} / L_{V}\right)$. 


\subsection{The mass-to-light ratio}

According to Section 1 and equation (7), it is clear that the dependence of $M / L$ on luminosity (or mass) is central to the task of building MFs from the observed LF. In this sense, we consider here the following comprehensive range of $M / L$ shapes: (i) constant: $\mathcal{M} / L_{V}=a$; (ii) linearly increasing with luminosity: $\mathcal{M} / L_{V}=a+b L_{V}$; (iii) power law: $\mathcal{M} / L_{V}=a+b L_{V}^{n}$; and (iv) $\operatorname{logarithmic:} \mathcal{M} / L_{V}=a+b \log \left(L_{V}\right)$, where $a$ and $b$ are constants. Note that for practical reasons (i.e. the need of an analytical expression to convert $M_{V}$ into $\mathcal{M}$ in equation 7), we express $M / L$ as a function of $L_{V}$ and not mass.

Obviously, once the $M / L$ constants $a$ and $b$ have been assigned values, the minimum and maximum present-day GC masses $\left(\mathcal{M}_{\mathrm{i}}\right.$ and $\mathcal{M}_{\mathrm{s}}$ ) are naturally determined from the respective faint and bright boundaries of the observed LF, and, for a given mean $\mathrm{GC}$ age $\left(\bar{t}_{\mathrm{gc}}\right)$, a similar reasoning leads to the maximum GC mass $\left(\mathcal{M}_{\text {sup }}\right)$ at $t_{\mathrm{o}}$ through inversion of equation (4).

\section{SEARCHING FOR THE OPTIMUM PARAMETERS}

The approach summarized by equation (7) contains some poorly known (and unknown) parameters, such as the mean cluster age $\left(\bar{t}_{\mathrm{gc}}\right)$, the dissolution time-scale $\left(t_{5}\right)$, the truncation mass $\left(\mathcal{M}_{\mathrm{c}}\right)$, and the $M / L$ constants $a$ and $b$. Our approach considers these five parameters as free. However, we note that some dependence between these parameters should be expected to occur, especially for very wide search ranges. For instance, a short $\bar{t}_{\mathrm{gc}}$ would result in a low $\tau_{5}$ to hasten dissolution over a shorter age range. Similarly, a lower $M / L$ would shift the MF turnover to a lower cluster mass, which could result from a longer dissolution time-scale (i.e. less dynamical evolution) for a given mean cluster age. We minimize this effect by adopting a reasonable GC age range (see below).

Thus, the task now is to search for values for the set of free parameters $\boldsymbol{P} \equiv\left\{a, b, \bar{t}_{\mathrm{gc}}, t_{5}, \mathcal{M}_{\mathrm{c}}\right\}$ which produce the best match between the observed and theoretical LFs, $\phi\left(M_{V}\right)$ and $\tilde{\phi}\left(M_{V}\right)$, respectively. This is achieved by minimizing the root mean squared residual $\left(R_{\mathrm{rms}}\right)$ between both functions. By construction, $R_{\mathrm{rms}}$ is a function of the free parameters, that is, $R_{\mathrm{rms}}=R_{\mathrm{rms}}(\boldsymbol{P})$, which we express as

$R_{\mathrm{rms}}=\sqrt{\frac{1}{N_{\mathrm{GC}}} \sum_{j=1}^{n_{\mathrm{b}}} \frac{\left[\tilde{\phi}\left(M_{V}^{j}\right)-\phi\left(M_{V}^{j}\right)\right]^{2}}{\tilde{\phi}\left(M_{V}^{j}\right)+\phi\left(M_{V}^{j}\right)}}$,

where the sum occurs over the $n_{\mathrm{b}}$ non-zero magnitude bins of the observed LF. The normalization by the theoretical+observed density of GCs in each bin gives a higher weight to the more populated bins. ${ }^{2}$ Finally, the squared sum is divided by the total number of observed GCs, which makes $R_{\mathrm{rms}}$ dimensionless and preserves the number statistics when comparing LFs built with unequal GC populations.

Among the several minimization methods available in the literature, the adaptive simulated annealing (ASA) is adequate to our purposes, because it is relatively time efficient, robust and capable to distinguish between different local minima (e.g. Goffe, Ferrier $\&$ Rogers 1994). The first step in the minimization process is to

${ }^{2}$ In Poisson statistics, where the uncertainty $\left(\sigma_{\phi}\right)$ of a signal $\phi$ is $\sigma_{\phi}=\sqrt{\phi}$, our definition of $R_{\mathrm{rms}}$ turns out being equivalent to the usual $\chi^{2}$. define individual search ranges (and variation steps) for each free parameter. Next, initial values are randomly selected for all free parameters (i.e. the initial point $\boldsymbol{P}_{\mathrm{o}}$ in the $R_{\mathrm{rms}}$ hypersurface) and the starting value of $R_{\mathrm{rms}}$ is computed. Then ASA takes a step by changing the initial parameters $\left(\boldsymbol{P}_{\mathrm{o}} \rightarrow \boldsymbol{P}\right)$, and a new value of $R_{\mathrm{rms}}$ is evaluated. Specifically, this implies that a new LF is built with the changed parameters. By definition, any step that decreases $R_{\text {rms }}$ (downhill) is accepted, with the process repeating from this new point. However, uphill steps may also be taken, with the decision made by the Metropolis (Metropolis et al. 1953) criterion, which has the advantage of enabling ASA to escape from local minima. The variation steps become smaller as the minimization is successful and ASA approaches the global minimum $\left(\boldsymbol{P} \rightarrow \boldsymbol{P}_{\min }\right)$.

The output of a single ASA run is the set of optimum values $\boldsymbol{P}_{\text {min }}$ (expected to correspond to the global minimum) and the respective $R_{\mathrm{rms}}$, but with no reference to uncertainties. However, given the errors associated with the observed LF (Fig. 1), uncertainties to the derived parameters are strongly required. Thus, we run ASA several times $\left(N_{\text {run }}\right)$ allowing for different starting points $\left(\boldsymbol{P}_{\mathrm{o}}\right)$ and taking the uncertainty of each $M_{V}$ bin in the observed LF (Fig. 1) into account. In the end, we compute the weighted mean of the parameters over a range of runs, using the $R_{\mathrm{rms}}$ of each run as weight ( $w=1 / R_{\mathrm{rms}}$ ). As a compromise between statistical significance and computational time, we adopt here $N_{\text {run }}=6000$.

The search ranges adopted here try to emulate the physical conditions prevailing in the Milky Way. In this sense, the mean GC age falls in the range $9 \leq \bar{t}_{\mathrm{gc}}(\mathrm{Gyr}) \leq 13$; the exponential truncation mass in the range $10^{4} \leq \mathcal{M}_{\mathrm{c}}\left(\mathrm{M}_{\odot}\right) \leq 10^{11}$; and the dissolution time-scale in the range $5 \leq \tau_{5}(\mathrm{Gyr}) \leq 30$ (to account for different environments and orbital conditions). Initially, the $M / L$ constants $a$ and $b$ were allowed to vary between 0 and 10, but, after a few runs, their ranges were fine-tuned to more constrained ranges.

\section{RESULTS AND DISCUSSION}

The approach described in Section 3 was applied to the four $M / L$ modes discussed in Section 2.1, and the numerical results of the 6000 independent ASA runs are summarized in Table 1. For conciseness, we show only the parameters belonging to the run with the absolute minimum $R_{\mathrm{rms}}$, together with those averaged over all the 6000 runs, and those corresponding to the average of 1 per cent of the runs having the lowest $R_{\mathrm{rms}}$. The LFs corresponding to the absolute minimum $R_{\mathrm{rms}}$ are compared to the observed LF in Fig. 1. Given the relative proximity between the minimum and average parameters (Table 1), the respective LFs of each $M / L$ should also be similar.

Qualitatively, the adopted $M / L$ s produce similarly evolved LFs (Fig. 1), but with different individual and integrated parameters at the output. As expected, the mean age, in all cases, is consistent with the age of the Galaxy and the age spread of the Galactic GCs (Muratov \& Gnedin 2010), ranging between 10 and $12.6 \mathrm{Gyr}$. Also, the best-fitting dissolution time-scale falls in the range $5 \leq$ $\tau_{5}(\mathrm{Gyr}) \leq 10$, a spread that is consistent with the different environments where the Galactic GCs dwell. Below we discuss the results for the $M / L$ modes in a decreasing order of $R_{\mathrm{rms}}$.

Before doing so, it is useful to gather some integrated Galactic $\mathrm{GC}$ values to be used for comparison with our results. For instance, based on the uniform ratio $\mathcal{M} / L_{V}=2$, Mackey \& van den Bergh (2005) estimate the current total mass of halo GCs to be $2 \times 10^{7} \mathrm{M}_{\odot}$. However, this may be a lower limit to the actual value of $M_{\mathrm{GC}}^{\text {now }}$, since halo GCs correspond to a subpopulation of the Galactic GCs (e.g. Mackey \& van den Bergh 2005) and single stellar population 
Table 1. PDMF and ICMF parameters found with alternative $M / L s$.

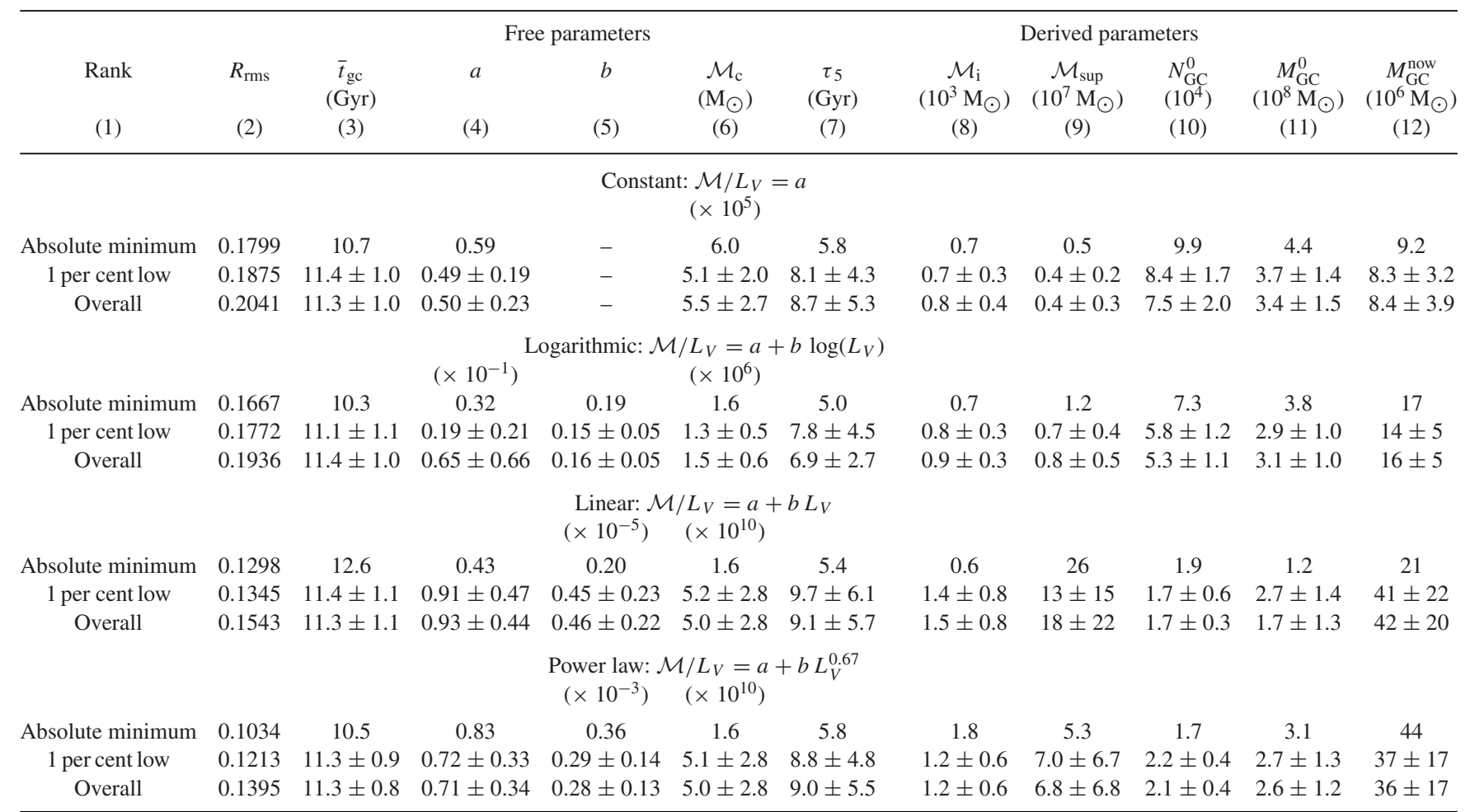

Notes. Columns (1) and (2): rank and corresponding $R_{\mathrm{rms}}$ value; Column (3): mean GC age; Columns (4) and (5): M/L constants; Column (6) exponential truncation mass; Column (7): dissolution time-scale of a $10^{5}-\mathrm{M}_{\odot}$ star cluster; Column (8): lowest present-day (observed) GC mass; Column (9): upper cluster mass at $t_{0}$; Columns (10) and (11): number and total mass of GCs at $t_{\mathrm{o}}$ (with $\mathcal{M}_{\text {inf }} \gtrsim 10^{2} \mathrm{M}_{\odot}$ ); Column (12): present-day total GC mass.

models (e.g. Anders \& Fritze-von Alvensleben 2003; Bruzual \& Charlot 2003) usually predict the higher ratio $\mathcal{M} / L_{V}=2-4$. Also, the initial total mass in GCs $\left(M_{\mathrm{GC}}^{0}\right)$ can be compared with estimates of the current stellar halo mass of $\mathcal{M}_{\mathrm{h}} \approx 1 \times 10^{9} \mathrm{M}_{\odot}$ (e.g. Freeman \& Bland-Hawthorn 2002).

Constant $\left[\mathcal{M} / L_{V}=a\right]$. With $R_{\mathrm{rms}} \sim 0.18-0.2$, the best fit was obtained with $\mathcal{M} / L_{V} \approx 0.6$. For such $M / L$, the lowest present-day GC mass turns out being somewhat low for a $\mathrm{GC}, \mathcal{M}_{\mathrm{i}} \sim 700 \mathrm{M}_{\odot}$; the exponential truncation mass is $\mathcal{M}_{\mathrm{c}} \approx 6 \times 10^{5} \mathrm{M}_{\odot}$. Reflecting the low statistical significance of the constant $M / L$, the dispersion around the mean for most parameters is very large. When combined, the derived parameters imply an exceedingly low (see above) total mass for the present-day GC population, $M_{\mathrm{GC}}^{\text {now }} \lesssim 1 \times 10^{7} \mathrm{M}_{\odot}$. Assuming $100 \mathrm{M}_{\odot}$ as the lower limit for cluster formation, the total stellar mass stored in clusters at $t_{\mathrm{o}}$ would be $M_{\mathrm{GC}}^{0} \sim 4 \times 10^{8} \mathrm{M}_{\odot}$. Thus, the fraction of the initial stellar mass still bound in GCs would be as low as $f_{\text {bound }} \approx 2$ per cent. The higher ratio $\mathcal{M} / L_{V}=2$ (the average used in previous works, e.g. Kruijssen 2008) leads to an evolved LF that fails to describe the observations, especially around the peak $\left(M_{V} \approx-7\right)$, with the high value of $R_{\mathrm{rms}} \sim 0.36$.

Logarithmic $\left[\mathcal{M} / L_{V}=a+b \log \left(L_{V}\right)\right]$. With $R_{\mathrm{rms}}$ similar to the constant $M / L$, the logarithmic $M / L$ yields $\mathcal{M}_{\mathrm{i}} \sim 800 \mathrm{M}_{\odot}, \mathcal{M}_{\mathrm{c}} \sim$ $1.5 \times 10^{6} \mathrm{M}_{\odot}, M_{\mathrm{GC}}^{\text {now }} \sim 1.5 \times 10^{7} \mathrm{M}_{\odot}$ and $M_{\mathrm{GC}}^{0} \sim 3 \times 10^{8} \mathrm{M}_{\odot}$, which implies the low bound mass fraction of $f_{\text {bound }} \sim 5$ per cent. These values are similar to those obtained with the constant $M / L$ and, as such, may not be realistic.

Linear $\left[\mathcal{M} / L_{V}=a+b L_{V}\right]$. Dropping somewhat to $R_{\mathrm{rms}} \sim$ $0.13-0.15$, the linearly increasing $M / L$ predicts the more realistic values of $\mathcal{M}_{\mathrm{i}} \sim 1.4 \times 10^{3} \mathrm{M}_{\odot}$ and $M_{\mathrm{GC}}^{\text {now }} \sim 4 \times 10^{7} \mathrm{M}_{\odot}$. With $M_{\mathrm{GC}}^{0} \sim 2 \times 10^{8} \mathrm{M}_{\odot}$, the fraction of stellar mass still bound in GCs rises to $f_{\text {bound }} \sim 20$ per cent. Interestingly, the best fits require an exponential truncation mass of $\mathcal{M}_{\mathrm{c}} \sim 10^{10} \mathrm{M}_{\odot}$, about four orders of magnitude higher than usually adopted (e.g. McLaughlin \& Fall 2008).

Power law $\left[\mathcal{M} / L_{V}=a+b L_{V}^{n}\right]$. To minimize the number of free parameters, we applied our approach for several fixed exponents $n$, with the lowest values of $R_{\text {rms }}(\sim 0.10-0.14)$ obtained with $n=0.67$. The relevant best-fitting parameters are $\mathcal{M}_{\mathrm{i}} \sim 10^{3} \mathrm{M}_{\odot}$, $\mathcal{M}_{\mathrm{c}} \sim 5 \times 10^{10} \mathrm{M}_{\odot}, M_{\mathrm{GC}}^{\text {now }} \sim 4 \times 10^{7} \mathrm{M}_{\odot}$ and $M_{\mathrm{GC}}^{0} \sim 3 \times 10^{8} \mathrm{M}_{\odot}$, with a bound mass fraction of $f_{\text {bound }} \sim 15$ per cent.

Both the linear and power-law models predict values of $M_{\mathrm{GC}}^{\text {now }}$ that are compatible with independent estimates. They also imply an initial total GC mass that corresponds to $\lesssim 30$ per cent of the current stellar halo mass.

Reflecting the (cluster) mass-dependent nature of the mass-loss processes, GCs having a mass in the range $(1-2) \times 10^{5} \mathrm{M}_{\odot}$ at the onset of the gas-free phase would have evolved to become the present-day least massive $\left(\mathcal{M}_{\mathrm{i}} \sim 1 \times 10^{3} \mathrm{M}_{\odot}\right)$ GCs. This means that, after a Hubble time of evolution in the Galaxy, GCs occupying the low-mass tail of the PDMF retain only $\sim 1$ per cent of their initial stellar mass. Regarding the total stellar mass still bound in GCs, the best-fitting $M / L$ s (power law and linear) imply that $\sim 85$ per cent of the mass of the initial GC population would have been lost to the field.

Finally, the best-fitting $M / L$ s are shown later in Fig. 3 as a function of cluster mass. Both the constant and logarithmic $M / L$ s are somewhat shallow, with $\mathcal{M} / L_{V} \lesssim 2.0$ over the full range of mass $\left[10^{2} \leq \mathcal{M}\left(\mathrm{M}_{\odot}\right) \leq 10^{7}\right]$. On the other hand, the linear and powerlaw $\bar{M} / L$ s have $\mathcal{M} / L_{V} \approx 1.0$ for $10^{2} \leq \mathcal{M}\left(\mathrm{M}_{\odot}\right) \lesssim 10^{4}$, then rising monotonically for larger masses, reaching $\mathcal{M} / L_{V} \approx 2.5$ for $\mathcal{M}=10^{6} \mathrm{M}_{\odot}$, and $\mathcal{M} / L_{V} \approx 4-8$ for $\mathcal{M}=10^{7} \mathrm{M}_{\odot}$. When converted to a function of GC mass, the power-law $M / L$ follows the 
relation

$\mathcal{M} / L_{V}=(0.78 \pm 0.01)+\left[\frac{\mathcal{M}}{(2.17 \pm 0.06) \times 10^{5} \mathrm{M}_{\odot}}\right]^{0.42 \pm 0.01}$.

In summary, the best results in terms of minimum $R_{\mathrm{rms}}$ residuals and realistic GC parameters are obtained with $M / L$ s that are relatively low for most of the GC mass range, but increase with luminosity (or mass), in the present case, expressed as a power law (exponent 0.67 ) or linearly.

\subsection{The PDMF}

One of the main results of our approach is to provide access to the PDMF through equation (5) and the best-fitting parameters of each $M / L$ mode. For practical reasons, they are shown in terms of $\mathrm{d} N / \mathrm{d} \log (\mathcal{M})$ in Fig. 2. They all clearly resemble a lognormal mass distribution, ${ }^{3}$ which is confirmed by the corresponding fit the parameters are given in Table 2. Again, the lognormal character applies especially to the MFs produced by the power-law and linear $M / L$ s, followed by the logarithmic and constant modes.

Overall, the PDMF mass turnover predicted by our $M / L$ s occurs in the range $\mathcal{M}_{\text {to }} \sim(3-8) \times 10^{4} \mathrm{M}_{\odot}$ (the upper bound corresponding to the power-law $M / L$ ), a range somewhat lower than the $\mathcal{M}_{\text {to }} \sim(1-2) \times 10^{5} \mathrm{M}_{\odot}$ range derived in previous works (e.g. McLaughlin \& Fall 2008). Probably, the difference occurs because for most of the GC mass range, our $M / L$ models are lower than $\mathcal{M} / L_{V} \simeq 2$ usually employed in previous studies, which naturally shifts $\mathcal{M}_{\text {to }}$ to lower values, implying longer dissolution time-scales. In addition, by not including the effects of shocks with giant molecular clouds and spiral arms, and two-body evaporation and ejection, ${ }^{4}$ equation (4) probably yields a softer mass-loss rate than those previously used. We also note that, for masses larger than that of the turnover, the PDMFs (of the power-law and linear $M / L s$ ) have a slope similar (within the uncertainties) to the MFs of young clusters and molecular clouds observed in the Milky Way and other galaxies (McLaughlin \& Fall 2008, and references therein). A similar high-mass slope was found for a sample of Galactic and M31 GCs by McLaughlin \& Pudritz (1996). Actually, this is a natural consequence of the large $\mathcal{M}_{\mathrm{c}}$, which favours the formation of a higher number of massive clusters with respect to a low- $\mathcal{M}_{\mathrm{c}} \mathrm{ICMF}$. Massive clusters are naturally long-lived and, thus, the slope of the large-mass tail of the ICMF tends to be preserved for longer periods of time.

We note that our best-fitting $M / L$ model predicts a maximum PDMF mass (Fig. 2) that is compatible with current estimates for Omega Centauri (NGC 5139): $2.5 \times 10^{6} \mathrm{M}_{\odot}$ (van de Ven et al. 2006), $4 \times 10^{6} \mathrm{M}_{\odot}\left(\right.$ Pryor \& Meylan 1993) and $5.1 \times 10^{6} \mathrm{M}_{\odot}$ (Meylan et al. 1995). In particular, the latter estimate implies the ratio $\mathcal{M} / L_{V} \approx 4$.1, again consistent with the power-law $M / L$ value for a GC with mass in the range $(2-5) \times 10^{6} \mathrm{M}_{\odot}$ (Fig. 3). The power-law $M / L$ predicts the mean present-day GC mass $\overline{\mathcal{M}} \approx$ $2.5 \times 10^{5} \mathrm{M}_{\odot}$, from which follows the scaling relation $\overline{\mathcal{M}} \sim 3 \mathcal{M}_{\mathrm{to}}$.

We also show in Fig. 2 the ICMFs reconstructed with the bestfitting parameters. Interestingly, while the ICMFs predicted by the constant and logarithmic $M / L$ s show the typical break at $\mathcal{M}_{\mathrm{c}} \sim 10^{5} \mathrm{M}_{\odot}$ of the Schechter profile, those of the linear and

\footnotetext{
${ }^{3}$ Expressed as $\frac{\mathrm{d} N}{\mathrm{~d} \log (\mathcal{M})} \propto \mathrm{e}^{-0.5\left[\frac{\log (\mathcal{M})-\overline{\log (\mathcal{M})}}{\sigma}\right]^{2}}$

${ }^{4}$ Although molecular clouds and spiral arms may not be relevant to most GCs, evaporation does play a role (e.g. McLaughlin \& Fall 2008).
}

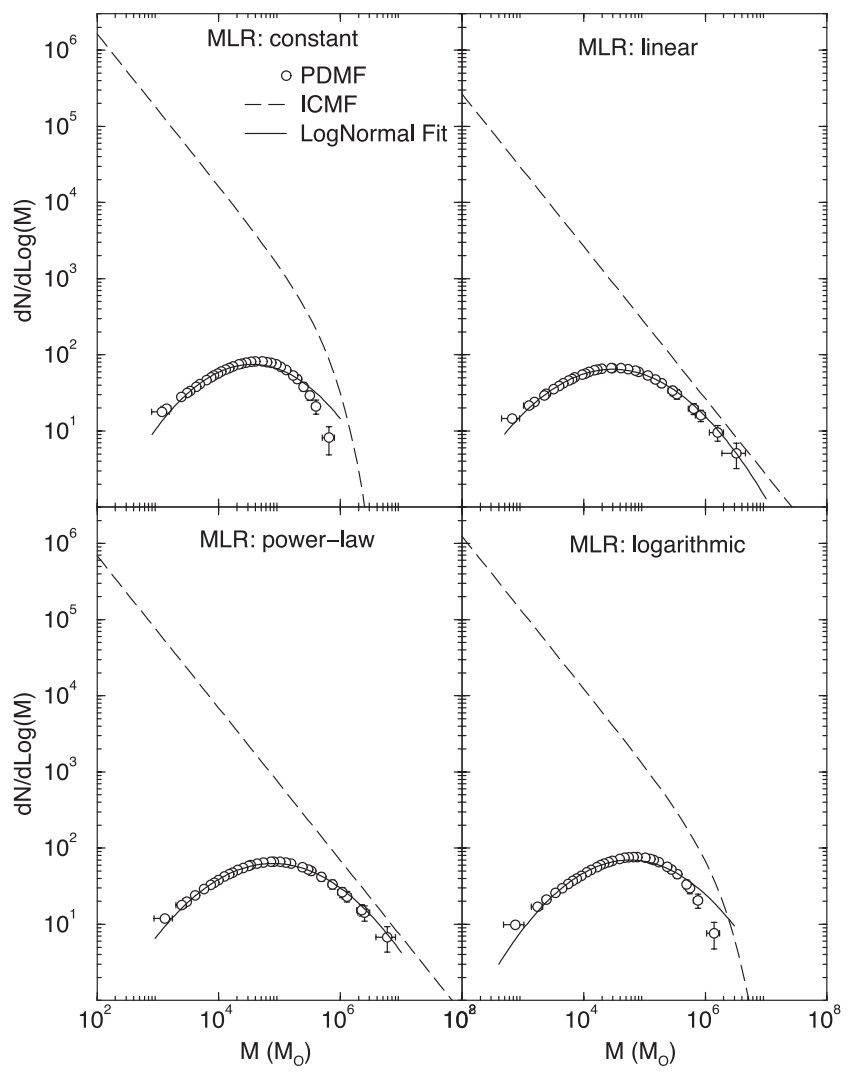

Figure 2. PDMFs (empty circles) and ICMFs (dashed line) that result from different $M / L s$. The PDMFs [expressed as $\mathrm{d} N / \mathrm{d} \log (\mathcal{M})$ ] are fitted with lognormal curves (thin solid line).

Table 2. Lognormal parameters of the PDMF.

\begin{tabular}{lccccc}
\hline$M / L$ & $\begin{array}{c}\mathcal{M}_{\text {to }} \\
\left(10^{4} \mathrm{M}_{\odot}\right)\end{array}$ & $\begin{array}{c}\sigma \\
\left(\mathrm{M}_{\odot}\right)\end{array}$ & $\mathrm{CC}$ & $\begin{array}{c}\overline{\mathcal{M}} \\
(3)\end{array}$ & $\overline{\mathcal{M}} / \mathcal{M}_{\text {to }}$ \\
$(1)$ & $(2)$ & $(4)$ & $(5)$ & $(6)$ \\
\hline Constant & $3.5 \pm 0.2$ & $0.80 \pm 0.02$ & 0.970 & 0.6 & 1.6 \\
Logarithmic & $5.7 \pm 0.4$ & $0.86 \pm 0.02$ & 0.989 & 1.0 & 1.8 \\
Linear & $3.1 \pm 0.2$ & $0.90 \pm 0.01$ & 0.997 & 2.7 & 8.6 \\
Power law & $7.8 \pm 0.2$ & $0.91 \pm 0.01$ & 0.997 & 2.5 & 3.2 \\
\hline
\end{tabular}

Notes: Column (2): turnover mass; Column (3): dispersion in $\log (\mathcal{M})$; Column (4): correlation coefficient of the lognormal fit to the PDMFs; Column (5): average GC mass; Column (6): ratio between the mean and turnover mass.

power-law $M / L$ s do not. Both are characterized by the large value of $\mathcal{M}_{\mathrm{c}} \sim 10^{10} \mathrm{M}_{\odot}$ and, thus, they end up resembling simply as a scale-free, power law MF of slope -1 (or -2 , when expressed as $\mathrm{d} N / \mathrm{d} \mathcal{M}$ ) over the very wide mass range $10^{2}-10^{10} \mathrm{M}_{\odot}$. The latter is a typical feature of young cluster MFs observed in nearby galaxies (e.g. Zhang \& Fall 1999; Portegies Zwart et al. 2010).

\section{SUMMARY AND CONCLUSIONS}

We present an approach to recover both the ICMF and PDMF of the Galactic GCs, having as a constraint the observed luminosity distribution. This is achieved by taking a few mass-loss processes into account and assuming an $M / L$ that is either constant or increases with luminosity linearly, as a power law or logarithmically. 


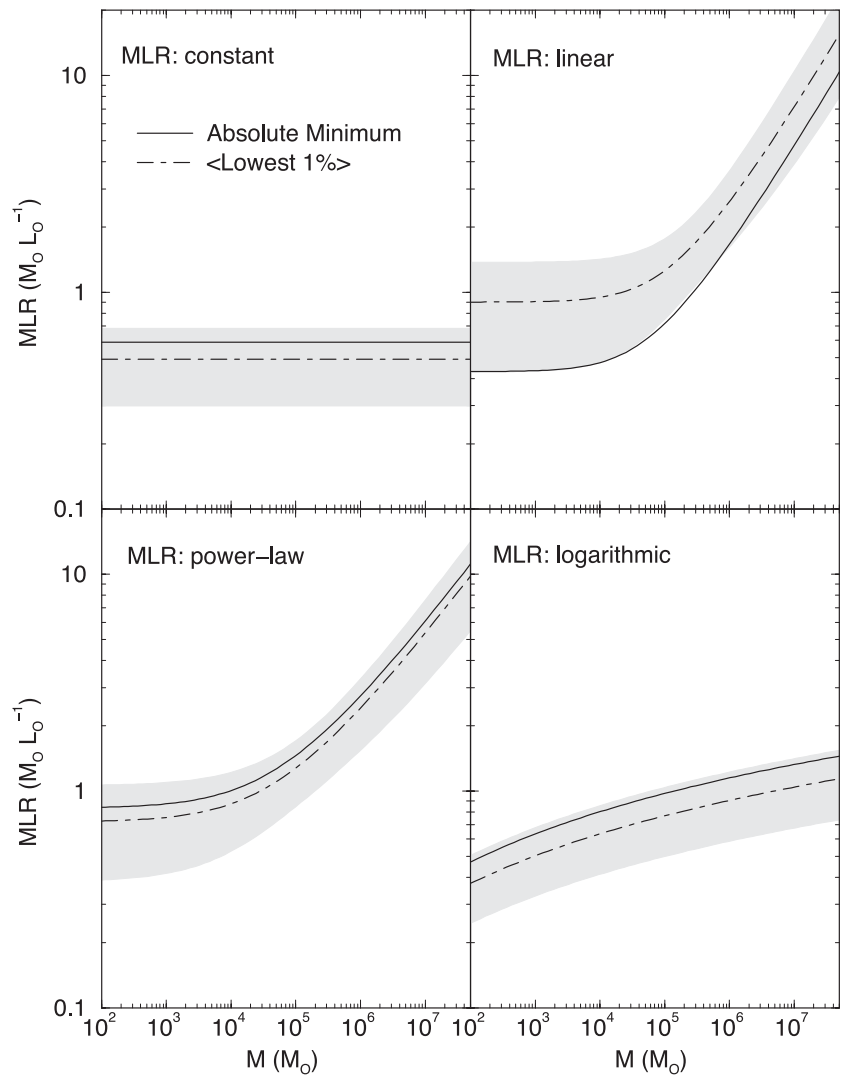

Figure 3. The best-fitting $M / L$ s are shown as a function of cluster mass. For comparison, the curves corresponding to the absolute minimum (solid line) and the 1 per cent runs with the lowest $R_{\text {rms }}$ values (dot-dashed line) are shown, together with the $1 \sigma$ uncertainty domain of the latter.

It starts with a Schechter-like ICMF, where the truncation $\left(\mathcal{M}_{\mathrm{c}}\right)$ mass is a free parameter. Then, using a set of parameters that usually apply to the dynamical dissolution of Galactic star clusters, the mean GC age $\left(\bar{t}_{\mathrm{gc}}\right)$, the dissolution time-scale of a $10^{5}-\mathrm{M}_{\odot}$ cluster $\left(\tau_{5}\right)$ and an $M / L$ (parametrized by the constants $a$ and $b$ ), we compute the MF shape after evolving for a time $\bar{t}_{\mathrm{gc}}$ after the onset of the gasfree phase. The evolved MF is subsequently converted into the LF. Finally, we search for the optimum parameters $\left(\bar{t}_{\mathrm{gc}}, t_{5}, \mathcal{M}_{c}, a, b\right)$ that minimize the residuals $\left(R_{\mathrm{rms}}\right)$ between the evolved and observed LFs.

The best results, both in terms of minimum values of $R_{\mathrm{rms}}$ and in terms of realistic parameters for the Galactic GCs, correspond to the power-law and linear $M / L$ s, respectively. Specifically, because both $M / L$ s increase with luminosity (or mass), their PDMFs imply a total mass in GCs of the order of $M_{\mathrm{GC}}^{\text {now }} \approx 4 \times 10^{7} \mathrm{M}_{\odot}$, which corresponds to a fraction of $\sim 15$ per cent of the initial stellar mass in GCs. On the other hand, the constant and logarithmic $M / L$ s are exceedingly shallow for the full GC mass range, yielding unrealistically low values of $M_{\mathrm{GC}}^{\text {now }}$.

Because of the large truncation mass $\left(\mathcal{M}_{\mathrm{c}} \sim 10^{10} \mathrm{M}_{\odot}\right)$, the Schechter-like ICMFs (of the linear and power-law $M / L$ s) end up resembling the scale-free, power-law MFs of young clusters and molecular clouds in the Milky Way and other galaxies. In addition, their PDMFs follow closely a lognormal distribution with a turnover mass at $\mathcal{M}_{\text {to }} \sim 7 \times 10^{4} \mathrm{M}_{\odot}$ which, for larger masses, behave as a power law with the same slope as the ICMFs. The low values of $\mathcal{M}_{\text {to }}$, compared to previous works, follow from the relatively low $M / L$ s (for most of the GC mass range) dealt with here.
Summarizing, it is clear that a power law is not a unique ICMF solution, the $M / L$, which serves to fine-tune the details, must increase with cluster mass (or luminosity), and that mass loss is the main factor responsible for shaping the ICMF into its present, lognormal form. Finally, our results suggest a common origin - in terms of physical processes - for GCs and young clusters.

\section{ACKNOWLEDGMENTS}

We thank an anonymous referee for relevant comments and suggestions. We acknowledge financial support from the Brazilian Institution CNPq.

\section{REFERENCES}

Anders P., Fritze-von Alvensleben U., 2003, A\&A, 401, 1063

Baumgardt H., 1998, A\&A, 330, 480

Baumgardt H., Kroupa P., 2007, MNRAS, 380, 1589

Baumgardt H., Kroupa P., Parmentier G., 2008, MNRAS, 384, 1231

Bica E., Bonatto C., Barbuy B., Ortolani S., 2006, A\&A, 450, 105

Bonatto C., Bica E., 2011, MNRAS, 415, 313

Bruzual G., Charlot S., 2003, MNRAS, 344, 1000

Elmegreen B. M., Falgarone E., 1996, ApJ, 471, 816

Freeman K., Bland-Hawthorn J., 2002, ARA\&A, 40, 487

Geyer M. P., Burkert A., 2001, MNRAS, 323, 988

Goffe W. L., Ferrier G. D., Rogers J., 1994, J. Econometrics, 60, 65

Goodwin S. P., 1997, MNRAS, 284, 785

Harris W. E., 1996, AJ, 112, 1487

Hilker M., Richtler T., 2000, A\&A, 362, 895

Kroupa P., Boily C. M., 2002, MNRAS, 336, 1188

Kruijssen J. M. D., 2008, A\&A, 486, L21

Kruijssen J. M. D., Cooper A. P., 2012, MNRAS, 420, 340

Kruijssen J. M. D., Portegies Zwart S. F., 2009, ApJ, 698, L158

Lada C. J., Margulis M., Dearborn D., 1984, ApJ, 285, 141

Lamers H. J. G. L. M., Gieles M., Bastian N., Baumgardt H., Kharchenko N. V., Portegies Zwart S., 2005, A\&A, 441, 117

Lamers H. J. G. L. M., Baumgardt H., Gieles M., 2010, MNRAS, 409, 305 Larsen S. S., 2009, A\&A, 494, 539

Mackey A. D., van den Bergh S., 2005, MNRAS, 360, 631

McLaughlin D. E., Fall S. M., 2008, ApJ, 679, 1272

McLaughlin D. E., Pudritz R. E., 1996, ApJ, 457, 578

Marín-Franch A. et al., 2009, ApJ, 694, 1498

Metropolis N., Rosenbluth A., Rosenbluth M., Teller A., Teller E., 1953, J. Chem. Phys., 21, 1087

Meylan G., Mayor M., Duquennoy A., Dubath P., 1995, A\&A, 303, 761

Muratov A. L., Gnedin O. Y., 2010, ApJ, 718, 1266

Parmentier G., Gilmore G., 2007, MNRAS, 377, 352

Parmentier G., Jehin E., Magain P., Noels A., Thoul A. A., 2000, A\&A, 363, 526

Parmentier G., Goodwin S. P., Kroupa P., Baumgardt H., 2008, ApJ, 678, 347

Portegies Zwart S. F., McMillan S. L. W., Gieles M., 2010, ARA\&A, 48, 431

Pryor C., Meylan G., 1993, in Djorgovski S. G., Meylan G., eds, ASP Conf. Ser. Vol. 50, Structure and Dynamics of Globular Clusters. Astron. Soc. Pac., San Francisco, p. 357

Schechter P., 1976, ApJ, 203, 297

Shin J., Kim S. S., Takahashi K., 2008, MNRAS, 386, L67

van de Ven G., van den Bosch R. C. E., Verolme E. K., de Zeeuw P. T., 2006, A\&A, 445, 513

Vesperini E., 1998, MNRAS, 299, 1019

Vesperini E., Zepf S. E., Kundu A., Ashman K. M., 2003, ApJ, 593, 760

Zhang Q., Fall S. M., 1999, ApJ, 527, L81

Zinn R., 1985, ApJ, 293, 424

This paper has been typeset from a $\mathrm{T}_{\mathrm{E}} \mathrm{X} / \mathrm{L} \mathrm{T} \mathrm{E} \mathrm{X}$ file prepared by the author. 